\title{
Biopharmaceutical innovation responsibility and ethical use of placebo
}

\begin{abstract}
Over the last era, the biopharmaceutical innovation has played an active role in health care and human life quality improvement. The contribution of new bio drugs in enhancing the population's health and providing welfare for the population in a sort that has never been seen before. However, despite all the benefits it has achieved, biopharmaceutical innovation does not escape from the bioethical challenge and rational guidelines that should adhere to its research process. Previously, the biopharmaceutical innovation was not deeply within the ethical debates Nevertheless, this character of thoughts has changed when the ethical standards awareness of the public and the health professionals have increased.
\end{abstract}

Nowadays, during testing new bio drugs and providing the patients with inactive treatment (A placebo masked in active treatment), ethical debates is noticeable and increasing. Indeed, new therapy techniques and placebo usage during the clinical research have not found convincing ethical answers yet. In addition, using the placebos has created many moral questions and gave birth to a big controversy in the health care field, in particular the misplace use of placebo in the research. The debate is deeper due to the fact that sometimes the clinicians instead of using the active therapeutic compounds, they use the placebos which might expose the patients to health risks. In this review, we give an ethical overview of the placebo use and its disadvantages in case of the misplaced use during the researches within the context of the biopharmaceutical responsible innovation.

Keywords: Placebo, Ethics, Biopharmaceutical innovation, Responsibility, Pharmacotherapies
Volume 5 Issue 6 - 2017

\author{
Kadour Ghanemi,' Shuangsheng Yan ${ }^{1,2}$ \\ 'Department of Business Management, School of International \\ Pharmaceutical Business, China Pharmaceutical University, \\ Nanjing, China \\ ${ }^{2}$ Department of Social Science, School of International \\ Pharmaceutical Business, China Pharmaceutical University, \\ Nanjing, China
}

\section{Correspondence: Kadour Ghanemi, Associate Professor, Director, the Philosophy of Teaching and Research Office, Department of Social Science, International Pharmaceutical Business School, China Pharmaceutical University, No 639, Longmian Avenue, Jiangning District, Nanjing, P.R. China, 21 I 198 , Tel 86 I33 05| 5 I7 82, Email ghanemikadour@outlook.com}

Received: July 23, 2017 | Published: August 24, 2017
Abbreviations:HIV, Human Immunodeficiency Virus; FDA, Food and Drug Administration

\section{Overview}

It is challenging to give a fixed definition and a special concept to describe the placebo, some are desperate to find such a specific concept for it. ${ }^{1}$ Many philosophers, scientists, and writers have a variety of opinions and positions about the use of placebo. ${ }^{2}$ Indeed, some of them see that it is not necessary to use placebo and consider it contradictory with the ethics of the health professions because it represents a deception for the patients, ${ }^{3,4}$ and it can also cause problems and care risk for them. ${ }^{5}$ In contrast, others see that it is not a kind of patient deception, but rather see the placebo as a white lie for the patients cure, and the physicians have no responsibility related to the way that the patients think about the research. ${ }^{6,7}$ In addition, the placebo might have satisfactory results, be efficient and benefits to the patients via avoiding risks. ${ }^{8,9}$

The placebo becomes a double-edged sword. It is a white lie to control and prevent the patients and, on the other hand, it exposes the patients to danger and risks. In most of the ethical guidelines and research standards, the misplaced placebo use is extremely unethical and can be harmful for patients and even lead to death.

\section{Placebo usage ethics:illustrative examples}

The closest concepts to the placebo define it as an ineffective and neutral compound which have no biologic actions and that the clinicians give to the patients as if it was an effective treatment, frequently the placebos are used (for instance) in the research trials so that physicians can do a comparison of the bio drug tested efficacy, in order to see whether that patients need a real bio drug or a placebo. ${ }^{10}$ Sometimes the physicians and researchers use the placebo in the clinical trials without informing the patients that the research is conducted through this kind of techniques and they might-somehowhide some of the expected risks that might happened, in other cases it is seen as having a negative impact on the study, the dilemma is that the researchers should have the agreement and the acceptance of the patients that the researcher is willing to use unreal therapy masked as a real therapy and accept the harm which might come out and even can lead patients to death. ${ }^{11-13}$ Moreover, hiding the truth from the patients and do not disclose the treatment method which the patient is undergoing is a violation of the patient rights because the patient wants an effective treatment but the doctor provides him with a placebo. ${ }^{14}$ This unethical behavior could be considered as a cheating deception and duplicity.

According to the declaration of Helsinki, the world medical association mentioned that the placebo can be used only in case when there is a lack of existing treatments, and the pharmacological candidate should be compared to the best drug available among the similar pharmacotherapies. ${ }^{15-18}$ This means that the use of the placebo is unacceptable in the case of the existence of an effective therapy. ${ }^{19,20}$ The world medical association publication of the declaration concerning the use of placebo was due to the big debate about the use of blinding method and placebo in the (acquired immune deficiency syndrome) AIDS research in Africa.

During an African study, covered eleven poor countries and sponsored and funded by different associations and developed countries, about testing the zidovudine (AZT) which prevent the transmission of the human immunodeficiency virus (HIV) from the pregnant women to her newborn, this preventative therapy costed 800 US dollars only for the AZT. ${ }^{21}$ Obviously, this therapy is 
affordable only in the developed countries and difficult to get in the developing countries where the HIV infection is getting widespread and becoming a huge dilemma, because the woman have various difficulties in addition to the limits in providing and affording the cost of AZT therapy. Based on this, the physicians used the placebo control and provided the women with a cheap and regiment therapy which is less efficient than the one used in the producing countries which is better and with more benefits than using no treatment. ${ }^{22,23}$ This research method gave birth to a lot of controversial ethical and responsible questions. For instance, since the main aim of the physicians researchers is to prevent the infants from being infected by the AIDS, why they are making the newborn vulnerable to AIDS infection by using an inefficacy therapy? In this case, the irresponsible behavior of the misplace using of the placebo is obvious. In fact, if the researchers used an active therapy instead of using the placebo, the rate that the baby will be infected would be reduced.

According to the Food and Drug Administration (FDA) policy, if the use of placebo can give harm and complicate the disease or lead to death and a treatment that can heal and prevent the disease exist, it is inappropriate to use the placebo. ${ }^{24}$ Furthermore, why the woman in the poor countries cannot be treated the same as the woman in developed countries and be provided with the real and the full-efficacy treatment during the research? According to the ethical rules and standards for health-related studies involving human subjects, during a research, the human subject in the developing countries should receive the same therapeutic method as in the sponsoring and developing countries, ${ }^{25,26}$ otherwise it is considered a part of racism. Building on this evidence, the misplaced use of the placebo is extremely unethical and irresponsible.

\section{Biopharmaceutical ethics and placebo}

The biopharmaceutical innovation is one of the most important concept within the medical innovation. With its new bio drugs, it is considered as a humanity relief. However, the biopharmaceutical innovation is not only about products, it is about ethical issues too because it is the ethics which define the origins that cause the patient harm or wellbeing. Thus, the ethics is a criterion for measuring the effectiveness and results of that bio drug innovation. Frequently, the companies innovate new bio drugs for the benefits of the company and secondary for the patients benefits. Sometimes, a company falls in a conflict of interest. Indeed, researchers should always consider the patients interests as the first priority, and the whole company staff should never build their profits and benefits on the harm of the patients. Moreover, they should reduce the harm exposure rather than boosting the company benefits. This way generates the spread of the high morals and behaviors within the researchers, companies and healthcare communities in general and thus, enhance the patients healthcare and life quality, gain the patients and the public satisfaction and appreciation, as well as pushing and opening the appetite for more innovation.

\section{Acknowledgments}

We acknowledge the Jiangsu Overseas Research \& Training Program for University Prominent Young \& Middle-aged Teachers. Kadour Ghanemi is the recipient of a full scholarship from the China Scholarship council. Abdelaziz Ghanemi provided partial assistance in revising the manuscript.

\section{Conflicts of interest}

The authors declare that there is no conflict of interest.

\section{References}

1. Gotzsche PC. Is there logic in the placebo? Lancet. 1994;344(8927):925926.

2. Shapiro AK. Attitudes toward the use of placebos in treatment. J Nerv Ment Dis. 1960;130:2OO-211.

3. Reiser SJ. Words as scalpels:Transmitting evidence in the clinical dialogue. Ann Intern Med. 1980;92(6):837-842.

4. Simmons B. Problems in deceptive medical procedures:An ethical and legal analysis of the administration of placebos. $J$ Med Ethics. 1978;4(4):172-181.

5. Nardini C. The ethics of clinical trials. E cancer medical science. 2014;8:387.

6. Mcdermott JF. A specific placebo effect encountered in the use of Dexedrine in hyperactive child. Am J Psychiatry. 1965;121:923-924.

7. Cassel C, Jameton AL. Power of the placebo:A dialog on principles and practice. Art Med. 1981;1(3):22-27.

8. Lichtenberg P, Heresco Levy U, Nitzan U. The ethics of the placebo in clinical practice. J Med Ethics. 2004;30(6):551-554.

9. Miller FG, Colloca L, Kaptchuk TJ. The placebo effect:illness and interpersonal healing. Perspect Bio Med. 2009;52(4):518-539.

10. McQuay HJ, Moore RA. Placebo. Postgrad Med J. 2005;81:155-160.

11. Anne Harrington. The placebo effect:An interdisciplinary exploration. Harvard University Press, Cambridge, USA, 1999; pp. 272.

12. Roberts, Laura Weiss. Placebos and paradoxes in psychiatric research:an ethics perspective. Biol Psychiatry. 2000;49(11):887-893.

13. Kadour Ghanemi, Shuangsheng Yan. Biopharmaceutical Innovation:Benefits and Challenges. Open Access Journal of Science. 2007;1(1):1-3.

14. De Deyn PP, Dhooge R. Placebos in clinical practice and research. $J$ Med Ethics. 1996;22(3):140-146.

15. Stang A, Hense HW, Jöckel KH, et al. Is It Always Unethical to Use a Placebo in a Clinical Trial? PLoS Med. 2005;2(3):e72.

16. Simon R. Are Placebo Controlled Clinical Trials Ethical or Needed When Alternative Treatment Exists? Ann Intern Med. 2000;133(6):474 475

17. La Vaque TJ, Rossiter T. The Ethical Use of Placebo Controls in Clinical Research:The Declaration of Helsinki. Applied Psychophysiology and Biofeedback. 2001;26(1):23-37.

18. Lau JTF, Mao J, Woo J. Ethical issues related to the use of placebo in clinical trials. Hong Kong Med J. 2000;9(3):192-198.

19. Freedman B, Weijer C, Glass KC. Placebo orthodoxy in clinical research I:empirical and methodological myths. J Law Med Ethics. 1996;24(3):243-251.

20. Freedman B, Glass KC, Weijer C. Placebo orthodoxy in clinical research II:ethical, legal, and regulatory myths. J Law Med Ethics. 1996;24(3):252-259.

21. Varmus H, Satcher D. Ethical complexities of conducting research in developing countries. N Engl J Med. 1997;337(14):1003-1005.

22. Bayer R. The debate over maternal fetal HIV transmission prevention trials in Africa, Asia, and the Caribbean:racist exploitation or exploitation of racism? Am J Public Health. 1998;88(4):567-570.

23. Lurie P, Wolfe SM. Unethical trials of interventions to reduce perinatal transmission of human immunodeficiency virus in developing countries. N Engl J Med. 1997;337(12):853-856. 
24. Young SN, Annable L. The ethics of placebo in clinical psychopharmacology:the urgent need for consistent regulation. $J$ Psychiatry Neurosci. 2002;27(5):319-321.

25. WHO. International ethical guidelines for biomedical research involving human subjects. The Council for International Organizations of Medical Sciences (CIOMS) in collaboration with the World Health Organization (WHO) 2016, Geneva, Switzerland, 2006;P. 64.
26. Shapiro HT, Meslin EM. Ethical issues in the design and conduct of clinical trials in developing countries. N Engl J Med. 2001;345(2):139142 . 